\title{
Streaming-potential phenomena in the thin-Debye-layer limit. Part 3. Shear-induced electroviscous repulsion
}

\author{
ORY SCHNITZER AND EHUD YARIV \\ ${ }^{1}$ Department of Mathematics, Imperial College London \\ Queen's Gate 180, London SW7 2AZ, United Kingdom \\ ${ }^{2}$ Department of Mathematics, Technion - Israel Institute of Technology \\ Haifa 32000, Israel
}

(Received 30 October 2015)

\begin{abstract}
We employ the moderate-Péclet-number macroscale model developed in part 2 of this sequence (Schnitzer, Frankel \& Yariv, J. Fluid Mech., vol. 704, 2012, pp. 109-136) towards the calculation of electroviscous forces on charged solid particles, engendered by an imposed relative motion between these particles and the electrolyte solution in which they are suspended. In particular, we are interested in the kinematic irreversibility of these forces, stemming from the diffusio-osmotic slip which accompanies the salt-concentration polarisation induced by that imposed motion. We illustrate the electroviscous irreversibility using two prototypic problems, one involving side-by-side sedimentation of two spherical particles, and the other involving a force-free spherical particle suspended in the vicinity of a planar wall and exposed to a simple shear flow. We focus upon the pertinent limit of near-contact configurations, where use of lubrication approximations provides closedform expressions for the leading-order lateral repulsion. In this approximation scheme the need to solve the advection-diffusion equation governing the salt-concentration polarisation is circumvented.
\end{abstract}

\section{Introduction}

Electrokinetic transport has to do with the interaction between electric fields and the relative motion between electrolyte solution and charged surfaces. As such, it can be conceptually decomposed into 'field-driven' phenomena, where applied electric fields or concentration gradients result in fluid motion, and 'motion-driven' phenomena, where an imposed flow leads to the formation of an electric field. The 'streaming-potential' mechanism underlying the latter has to do with charge conservation: by sweeping charged fluid elements within the diffuse-charge layers adjacent to the charged surfaces, the imposed flow results in effective 'surface' currents, which are generally nonuniform. Charge conservation then necessities Ohmic charging from the surrounding electro-neutral bulk; since the bulk domain is essentially Ohmic, this implies the formation of a bulk electric field.

The rich physical phenomena associated with motion-driven electrokinetics have to do with the generation of forces by the induced electric field, above and beyond the hydrodynamic forces associated with the driving flow. These 'electroviscous' forces are contributed, on the one hand, by the Maxwell stresses accompanying any electric-field distribution, and, on the other, by a secondary electrokinetic flow driven by the action of the distributed field on the diffuse-charge layer. As electroviscous forces are in general nonlinear in the applied flow, their effect is not captured by the common linear-response 
approach, which views the connection between field-driven and motion-driven electrokinetic phenomena as a special case of the Onsager reciprocal relation (Doi \& Makino 2008).

Studies of electroviscous forces have focused upon such prototypic problems as the excess flow-resistance of narrow capillaries and slits (Elton 1948; Rice \& Whitehead 1965; Bowen \& Jenner 1995), the excess drag on sedimenting particles (Elton 1949; Booth 1954; Stigter 1980; Ohshima et al. 1984), and the enhanced viscosity of dilute suspensions (Booth 1950; Russel 1976, 1978b, a; Lever 1979; Watterson \& White 1981; Hinch \& Sherwood 1983). Electroviscous drag on particles approaching solid surfaces is in particular relevant to the prediction of coagulation and deposition rates of charged colloids (Warszynski \& van de Ven 1990, 1991).

It may appear from the above list of research problems that electroviscous forces essentially tend to retard the driving motion. These forces may play however an additional role by inducing motion perpendicular to the driving motion. Perhaps the most familiar illustration of this effect is non-inertial shear-induced respulsion ('electrokinetic lift'). This phenomenon was discovered by Alexander \& Prieve (1987) during an attempt to measure colloidal forces between microns-sized polystyrene particles and a solid surface. Alexander \& Prieve (1987) observed motion of the particles away from the wall at a rate which depends on an applied shear flow parallel to the wall, in contradiction with the linearity and reversibility properties of Stokes flow (Jeffrey 1996; Leal 2007). Since the most prominent effect was observed in glycerol, a highly viscous liquid, an inertial mechanism was discounted; rather, the observed attenuation with increasing ionic conductivity has suggested a linkage with streaming-potential phenomena.

The above phenomenon of electrokinetic lift exemplifies the typical breakdown of Stokes-flow reversibility brought about by electroviscous forces. These forces are necessarily nonlinear in the applied motion, or flow, and hence are not captured by low-Pécletnumber linearisations (Ohshima et al. 1984). A convenient path for studying nonlinear electroviscous phenomena is based on 'macroscale' descriptions in the thin-double-layer limit, where the Debye length is small compared to particle size. Such coarse-grained descriptions were derived in the first two parts of this series (Yariv, Schnitzer \& Frankel 2011; Schnitzer, Frankel \& Yariv 2012b) using a matched-asymptotics reduction of the standard 'microscale' electrokinetic equation set. The resulting models consist of a simplified set of equations governing the approximately electro-neutral bulk domain, along with a set of effective boundary conditions representing the transport within the diffuse-charge Debye layers. Together, parts 1 and 2 cover the entire range of the forcing intensities, viz. from low to very large Péclet numbers. As thoroughly discussed in these papers, our approach builds on earlier thin-double-layer models (Bike \& Prieve 1990; van de Ven et al. 1993; Bike \& Prieve 1995; Cox 1997; Warszynski et al. 1998), in effect resolving the controversy in the literature regarding the appropriate macroscale description in the mathematically singular thin-double-layer limit.

The models derived in parts 1 and 2 reveal that the physical mechanism responsible for kinematic irreversibility is essentially different at moderate and large Péclet numbers. Thus, in the large-Péclet-number régime considered in part 1, electroviscous forces originate due to both Maxwell stresses and the Newtonian stresses accompanying the secondary electrokinetic flow. The latter, however, being purely electro-osmotic, are linear and homogenous in the driving flow. Irreversibility is then triggered by the electric forces alone. The situation is rather different in the moderate-Péclet number model developed in part 2 of this sequence. In that limit, the Maxwell stresses do not affect the leading electroviscous forces. On the other hand, the secondary electrokinetic flow is now driven by both electro-osmosis and diffusio-osmosis, the latter engendered by an induced salt- 
concentration polarisation. Since this polarisation is governed by an advection-diffusion equation, diffusio-osmosis is inhomogeneous in the driving flow. Kinematic irreversibility thus stems from the Newtonian stresses associated with the diffusio-osmotic component of the secondary electrokinetic flow.

Using the model developed in part 1, we have illustrated large-Péclet-number irreversibility using two prototypical problems: one involving two spheres sedimenting sideby-side, where electroviscous forces result in a repulsion force along the line of centres (Schnitzer, Khair \& Yariv 2011), and one involving a particle in a shear flow (Schnitzer, Frankel \& Yariv 2012a). In the large-Péclet-number scheme, extracting the irreversible part of the electroviscous force is relatively straightforwardly since this part is solely associated with the induced electric field (the 'streaming potential').

While the simplicity of the large-Péclet-number scheme of part 1 is attractive, most relevant problems are characterised by moderate Péclet numbers. In particular, due to the nearly matching solid-liquid densities in the common case of polystyrene particles in aqueous solutions, typical Péclet numbers in sedimentation experiments are actually small. In problems involving imposed shear it is possible in principle to reach large Péclet numbers, but this was not the case in the experiments of Alexander \& Prieve (1987).

The goal of this paper is accordingly to illustrate kinematic irreversibility using the moderate-Péclet-number model of part 2. We consider the same prototypic problems studied with the large-Péclet-number model, namely particle-pair sedimentation and shear-induced lift. While shear is externally imposed only in the second problem, the irreversible electroviscous repulsion in both problems has to do with the local shear within the gap region (between the two particles in the first problem, and between the particle and the wall in the second). This shear is triggered by the relative motion of the rigid surfaces bounding the gap.

In both of the above problems, the advection-diffusion equation governing the saltconcentration polarisation cannot be solved in closed form. An analytic investigation of the above two problems thus appears intractable. Nonetheless, by focusing upon the common situation wherein the gap is narrow, we obtain useful lubrication-type approximations - a route already taken in the context of earlier thin-double-layer models (Bike \& Prieve 1990; van de Ven, Warszynski \& Dukhin 1993; Tabatabaei, van de Ven \& Rey 2006), and in our own demonstrations (Schnitzer et al. 2011, 2012a) of the large-Pécletnumber model of part 1 . As will become evident, the resulting approximations predict electroviscous forces that scale inversely with the square of the gap thickness. Thus, the narrow-gap limit highlights the very régime wherein irreversibility is most pronounced.

The paper is structured as follows. In the next section we recapitulate the moderatePéclet-number macroscale model. In $\S 3$ we discuss quasi-steadiness and the source of nonlinear irreversibility. In $\S 4$ we formulate the problems of particle-pair sedimentation and particle under shear. In $\S 5$ we set the stage for the lubrication analysis in the nearcontact limit upon which we focus. In $\S 6$ and $\S 7$ we respectively analyse the salt-transport problem and the consequent diffusio-osmotic flow. In $\S 8$ we calculate the irreversible repulsive force for both problems. In $\S 9$ we review our results, provide them in dimensional form, and discuss the linkage of the present analysis of shear-induced lift with that of Tabatabaei et al. (2006). 


\section{Recapitulation of the generic macroscale description}

\subsection{Generic problem and nondimensonalization}

Consider a generic force- or flow-driven electrokinetic problem, involving the motion of one or more charged solid surfaces (fixed surface-charge density $\sigma^{*}$ ) relative to an electrolyte solution (dielectric permittivity $\epsilon^{*}$, Newtonian viscosity $\mu^{*}$ ). For simplicity, we restrict our attention to a symmetric binary electrolyte solution (ionic valencies $\pm \mathscr{Z}$ ) and assume identical diffusivity $D^{*}$ of the two ionic species. The imposed flow or force is characterized by the velocity $v^{*}$ and the length scale $a^{*}$. An additional length scale is the Debye width $1 / \kappa^{*}$, defined by

$$
\kappa^{* 2}=\frac{2 \mathscr{Z} e^{*} c^{*}}{\epsilon^{*} \varphi^{*}}
$$

in which

$$
\varphi^{*}=\frac{k^{*} T^{*}}{\mathscr{Z} e^{*}}
$$

is the thermal voltage, wherein $k^{*} T^{*}$ is the Boltzmann temperature and $e^{*}$ the elementary charge. As in prototypic force- or flow-driven electrokinetic phenomena, no electric field or salt gradients are imposed. Thus, at large distances away from the solid surfaces the electric field vanishes and the two ionic concentrations approach their equilibrium value, say $c^{*}$.

We employ a dimensionless notation, normalizing length variables by $a^{*}$, velocities by $v^{*}$, and electric potentials by $\varphi^{*}$. Consistent with the above, we normalise angular velocities by $v^{*} / a^{*}$, stresses by $\mu^{*} v^{*} / a^{*}$, forces by $\mu^{*} v^{*} a^{*}$, and torques by $\mu^{*} v^{*} a^{* 2}$. The electrokinetic problem is governed by three key parameters: the first,

$$
\delta=\frac{1}{\kappa^{*} a^{*}},
$$

is the ratio of the Debye thickness and characteristic length-scale of the problem; the second is the Péclet number

$$
P e=\frac{a^{*} v^{*}}{D^{*}}
$$

and the third is the Hartmann number

$$
\lambda=\frac{2 k^{*} T^{*} a^{*} c^{*}}{\mu^{*} v^{*}} .
$$

Yariv et al. (2011) noted that the above three parameters are not independent, but are rather related as

in which

$$
\lambda P e=\frac{\alpha}{\delta^{2}},
$$

$$
\alpha=\frac{\epsilon^{*} \varphi^{* 2}}{\mu^{*} D^{*}}
$$

(which may represent a Péclet number in field-driven phenomena, see Saville 1977) is independent of both particle dimension $a^{*}$ and electrolyte concentration $c^{*}$. For typical ionic diffusivities $\left(D^{*} \approx 10^{-9} \mathrm{~m}^{2} \mathrm{~s}^{-1}\right)$ in aqueous solutions $\left(\mu^{*} \approx 10^{-3} \mathrm{~kg} \mathrm{~m}^{-1} \mathrm{~s}^{-1}\right), \alpha \lesssim$ 0.5. By the Einstein-Smoluchowski relation (2.7) is independent of $\mu^{*}$ and is accordingly of order unity for highly viscous solutions as well.

The identification of (2.6) implies that the thin-doubler-layer limit $\delta \ll 1$ can be studied using a family of different limit processes. In what follows we consider the (realistic) 
limit where the Péclet number is assumed moderate, as discussed in detail by Schnitzer et al. $(2012 b)$.

\subsection{Macroscale description}

We use the generic macroscale model developed by Schnitzer et al. (2012b), valid for $O(1)$ Péclet numbers. In that model, the pertinent bulk variables are the electric potential $\varphi$, the mean ('salt') ionic concentration $c$, and the flow field $\boldsymbol{v}$. The first two possess the following asymptotic expansions,

$$
\varphi=\delta^{2} \varphi_{2}+\cdots, \quad c=1+\delta^{2} c_{2}+\cdots,
$$

representing the weak disturbance at moderate $P e$. The flow field $\boldsymbol{v}$ is expanded as

$$
\boldsymbol{v}=\boldsymbol{v}_{0}+\delta^{2} \boldsymbol{v}_{2}+\cdots,
$$

with a similar expansion governing both the associated pressure field $p$

$$
p=p_{0}+\delta^{2} p_{2}+\cdots,
$$

and the surface-velocity of the bounding walls

$$
\boldsymbol{u}=\boldsymbol{u}_{0}+\delta^{2} \boldsymbol{u}_{2}+\cdots .
$$

Since these rigid walls undergo rigid-body motion, the latter amounts to an asymptotic expansion of their respective rectilinear and angular velocities. Finally, consider the Newtonian stress

$$
N=-p \boldsymbol{I}+(\boldsymbol{\nabla} \boldsymbol{v})+(\boldsymbol{\nabla} \boldsymbol{v})^{\dagger}
$$

in which $\dagger$ denotes tensor transposition. Since it is linear in $p$ and $\boldsymbol{v}$, it also possesses a similar expansion,

The scheme of Schnitzer et al. (2012b) is expressed in terms of the preceding expansions. The effective boundary conditions in that scheme, applied over the generic boundary $s$, represent a lumped macroscale description of the Debye-layer physics. In that description, the dimensionless surface-charge density $\sigma=\sigma^{*} / \epsilon^{*} \kappa^{*} \varphi^{*}$ on the solid wall is represented by the dimensionless zeta-potential $\zeta$ :

$$
\sigma=2 \sinh \frac{\zeta}{2}
$$

Consistently with the scheme of Schnitzer et al. (2012b), we assume that $\sigma$ (and then also $\zeta$ ) is uniform on each surface. It should be noted that $\zeta$ refers here to the Debye-layer voltage; the asymptotically small concentration polarisation (see $(2.8 b)$ ) results in a small perturbation to that voltage, but this has no bearing on the leading-order electrokinetic flow (Schnitzer et al. 2012b).

\subsection{Driving flow}

The leading-order flow $\left(\boldsymbol{v}_{0}, p_{0}\right)$ is governed by the continuity and homogenous Stokes equations,

$$
\boldsymbol{\nabla} \cdot \boldsymbol{v}_{0}=0, \quad \nabla p_{0}=\nabla^{2} \boldsymbol{v}_{0}
$$

together with the no-slip boundary condition on the solid boundaries,

$$
\boldsymbol{v}_{0}=\boldsymbol{u}_{0} \quad \text { on } s .
$$

In addition, at large distances it approaches a prescribed Stokes flow, say $\boldsymbol{v}_{\infty}(\boldsymbol{x})$ (wherein $\boldsymbol{x}$ denotes the position vector):

$$
\boldsymbol{v}_{0} \sim \boldsymbol{v}_{\infty}(\boldsymbol{x}) \quad \text { as } \quad|\boldsymbol{x}| \rightarrow \infty .
$$


The above leading-order flow is not affected by electrokinetic phenomena. In fact, it is just the flow field in the absence of surface charge. We accordingly refer to it as the 'driving' flow. Its leading-order correction $\boldsymbol{v}_{2}$, which would trivially vanish in the absence of surface charge, is thus denoted the 'electrokinetic' flow.

\subsection{Streaming potential and salt polarisation}

Considering $\boldsymbol{v}_{0}$ as known, the streaming-potential $\varphi_{2}$ and salt-concentration perturbation $c_{2}$ turn out to satisfy linear boundary-value problems. The streaming potential is governed by Laplace's equation in the fluid domain,

$$
\nabla^{2} \varphi_{2}=0,
$$

the far-field attenuation condition,

$$
\boldsymbol{\nabla} \varphi_{2} \rightarrow 0 \quad \text { as } \quad|\boldsymbol{x}| \rightarrow \infty,
$$

and the inhomogeneous Neumann condition on the solid boundaries

$$
\frac{\partial \varphi_{2}}{\partial n}=\zeta P e \frac{\partial p_{0}}{\partial n} \quad \text { on } \quad s,
$$

wherein $\partial / \partial n=\hat{\boldsymbol{n}} \cdot \boldsymbol{\nabla}$, in which $\hat{\boldsymbol{n}}$ is a unit vector normal to $s$, pointing into the fluid domain. The salt perturbation is governed by the advection-diffusion equation

$$
\nabla^{2} c_{2}=P e\left(\frac{\partial c_{2}}{\partial t}+\boldsymbol{v}_{\mathbf{0}} \cdot \nabla c_{2}\right),
$$

the inhomogeneous Neumann condition

$$
\frac{\partial c_{2}}{\partial n}=-\mathscr{H}(\zeta) P e \frac{\partial p_{0}}{\partial n} \quad \text { on } \quad s,
$$

and the attenuation condition

$$
c_{2} \rightarrow 0 \quad \text { as } \quad|x| \rightarrow \infty .
$$

The quantity

$$
\mathscr{H}(\zeta)=-2 \ln \left(1-\tanh ^{2} \frac{\zeta}{4}\right),
$$

appearing in (2.21), represents the 'salt capacitance' of the Debye layer. Note that the normal derivative of $p_{0}$ is proportional to the surface divergence of the tangential shear associated with $\boldsymbol{v}_{0}$; the effective conditions (2.19) and (2.21) thus represent the appropriate balances between Debye-scale advection of charge and salt and their corresponding bulk fluxes (Schnitzer et al. 2012b).

\subsection{Electrokinetic flow}

Conveniently, both the streaming potential and concentration polarisation are independent of the electrokinetic flow $\left(\boldsymbol{v}_{2}, p_{2}\right)$. Once $\varphi_{2}$ and $c_{2}$ are determined, $\left(\boldsymbol{v}_{2}, p_{2}\right)$ is obtained by solving an electro-diffusio-osmotic problem, consisting of the homogeneous Stokes equations

$$
\boldsymbol{\nabla} \cdot \boldsymbol{v}_{2}=0, \quad \boldsymbol{\nabla} p_{2}=\nabla^{2} \boldsymbol{v}_{2},
$$

and attenuation requirement

$$
\boldsymbol{v}_{2} \rightarrow \mathbf{0} \quad \text { as } \quad|\boldsymbol{x}| \rightarrow \infty,
$$


together with the slip condition

$$
\boldsymbol{v}_{2}-\boldsymbol{u}_{2}=\frac{\alpha}{P e}\left\{\zeta \nabla_{s} \varphi_{2}-\mathscr{H}(\zeta) \nabla_{s} c_{2}\right\} \quad \text { on } s,
$$

wherein

$$
\nabla_{s}=(I-\hat{\boldsymbol{n}} \hat{\boldsymbol{n}}) \cdot \nabla
$$

is the surface-gradient operator.

It is convenient to decompose $\boldsymbol{v}_{2}$ as

$$
\boldsymbol{v}_{2}=\boldsymbol{v}_{2, \boldsymbol{u}}+\boldsymbol{v}_{2, \varphi}+\boldsymbol{v}_{2, c}
$$

(with a similar decomposition for $p_{2}$ ), where each of the three components separately satisfies the homogenous equations (2.24) and the decay condition (2.25). On the boundary $s$, they respectively satisfy

$$
\boldsymbol{v}_{2, \boldsymbol{u}}=\boldsymbol{u}_{2}, \quad \boldsymbol{v}_{2, \varphi}=\frac{\alpha}{P e} \zeta \nabla_{s} \varphi_{2}, \quad \boldsymbol{v}_{2, c}=-\frac{\alpha}{P e} \mathscr{H}(\zeta) \nabla_{s} c_{2} .
$$

\subsection{Forces and torques}

In a typical situation the boundary $s$ includes the surface of one or more freely suspended particles. Given the flow-field expansion (2.9), similar expansions apply to both the hydrodynamic force $\boldsymbol{F}$ and torque $\boldsymbol{T}$ (about a reference point $\boldsymbol{x}_{O}$ ) acting on such a particle:

$$
\boldsymbol{F}=\boldsymbol{F}_{0}+\delta^{2} \boldsymbol{F}_{2}+\cdots, \quad \boldsymbol{T}=\boldsymbol{T}_{0}+\delta^{2} \boldsymbol{T}_{2}+\cdots .
$$

The zeroth-order force and torque

$$
\boldsymbol{F}_{0}=\oint \mathrm{d} A \hat{\boldsymbol{n}} \cdot N_{0}, \quad \boldsymbol{T}_{0}=\oint \mathrm{d} A\left(\boldsymbol{x}-\boldsymbol{x}_{O}\right) \times \hat{\boldsymbol{n}} \cdot N_{0},
$$

represent the hydrodynamic loads in the absence of electrokinetic effects. The electroviscous force and torque are similarly given by

$$
\boldsymbol{F}_{2}=\oint \mathrm{d} A \hat{\boldsymbol{n}} \cdot N_{2}, \quad \boldsymbol{T}_{2}=\oint \mathrm{d} A\left(\boldsymbol{x}-\boldsymbol{x}_{O}\right) \times \hat{\boldsymbol{n}} \cdot N_{2},
$$

where $N_{2}$ is the Newtonian stress associated with the electrokinetic flow $\boldsymbol{v}_{2}$. Note that the contribution of Maxwell stresses enters only at $O\left(\delta^{4}\right)$, and is accordingly disregarded in our analysis. This is in sharp contradiction to the case of large Péclet numbers discussed by Yariv et al. (2011).

The motion of the particle is determined by the conditions

$$
\boldsymbol{F}+\boldsymbol{F}_{e}=\mathbf{0}, \quad \boldsymbol{T}+\boldsymbol{T}_{e}=\mathbf{0},
$$

where $\boldsymbol{F}_{e}$ is the external force and $\boldsymbol{T}_{e}$ the external torque. In particular, the leading-order rigid-body motion of the particle (namely $\boldsymbol{u}_{0}$ ) is governed by the mobility problem,

$$
\boldsymbol{F}_{0}+\boldsymbol{F}_{e}=\mathbf{0}, \quad \boldsymbol{T}_{0}+\boldsymbol{T}_{e}=\mathbf{0} .
$$

The electroviscous correction is also governed by a mobility problem, namely

$$
\boldsymbol{F}_{2}=\mathbf{0}, \quad \boldsymbol{T}_{2}=\mathbf{0} .
$$

The decomposition (2.28) implies a similar one for $\boldsymbol{N}_{2}$ and hence also for $\boldsymbol{F}_{2}$ and $\boldsymbol{T}_{2}$. Since $\boldsymbol{F}_{2, \boldsymbol{u}}$ and $\boldsymbol{T}_{2, \boldsymbol{u}}$ simply represent the hydrodynamic force and torque associated with the rigid-body motion $\boldsymbol{u}_{2}$, the force $\boldsymbol{F}_{2, \varphi}+\boldsymbol{F}_{2, c}$ and torque $\boldsymbol{T}_{2, \varphi}+\boldsymbol{T}_{2, c}$ can be interpreted as 'external' loads. In what follows we focus on the calculation of these electroviscous loads. 


\section{Quasi-steadiness and irreversibility}

The preceding macroscale model applies to a generic motion-driven electrokinetic problem. The animating motion may be driven by either imposed flow, as appearing in (2.16), or imposed forces, as appearing in (2.33). In what follows, we illustrate our scheme for two representative problems. The first, driven by an imposed force, is that of two particles sedimenting side by side. The second, driven by an imposed flow, is that of a particle which is exposed to a simple shear flow in the vicinity of a planar wall.

In both of these problems, the reversibility properties of the Stokes flow (Leal 2007) guarantee the existence of a reference frame in which the flow is steady in the absence of electrokinetic effects. In that frame, time dependence enters through the irreversible $O\left(\delta^{2}\right)$ motion. The temporal-derivative term in $(2.20)$ is of relative order $\delta^{2}$, and is accordingly relegated to the next asymptotic balance. Thus, both the $O(1)$ flow problem and the $O\left(\delta^{2}\right)$ electrokinetic problems are quasi-steady, with time dependence entering only implicitly through the slow temporal variation of the problem geometry.

In this quasi-steady description, it is evident from the problem formulation that the streaming potential must be of the form

$$
\varphi_{2}(\boldsymbol{x} ; \operatorname{Pe}, \zeta)=\operatorname{Pe} \zeta \tilde{\varphi}(\boldsymbol{x}),
$$

where $\tilde{\varphi}$ is linear and homogeneous in the driving flow $\boldsymbol{v}_{0}$. The same form does not apply for the salt polarisation: while $c_{2}$ is governed by a linear boundary-value problem, it is evident from (2.20) that it is not homogenous in the driving flow. The perturbation here is therefore of the more general form

$$
c_{2}(\boldsymbol{x} ; P e, \zeta)=P e \mathscr{H}(\zeta) \tilde{c}(\boldsymbol{x} ; P e),
$$

where $\tilde{c}$ is in general nonlinear in the driving flow. This rescaled perturbation satisfies the differential equation

$$
\nabla^{2} \tilde{c}=P e \boldsymbol{v}_{\mathbf{0}} \cdot \nabla \tilde{c}
$$

the inhomogeneous Neumann condition

$$
\frac{\partial \tilde{c}}{\partial n}=-\frac{\partial p_{0}}{\partial n} \quad \text { on } \quad s
$$

and the far-field attenuation condition

$$
\tilde{c} \rightarrow 0 \quad \text { as } \quad|\boldsymbol{x}| \rightarrow \infty .
$$

By a straightforward extension of the reversibility properties of Stokes flows (Jeffrey 1996) it is readily seen that a Stokes flow that is linear in a prescribed forcing cannot result in irreversible particle motion. The streaming potential $\varphi$ is linear in the imposed flow $\boldsymbol{v}_{0}$, which itself is linear in the mechanical forcing; it then follows that the loads $\boldsymbol{F}_{2, \varphi}$ and $\boldsymbol{T}_{2, \varphi}$, associated with the 'electro-osmotic' component $\boldsymbol{v}_{2, \varphi}$ of $\boldsymbol{v}_{2}$, do not result in an irreversible motion. The same argument does not hold for the loads created by salt polarisation: since $\boldsymbol{v}_{2, c}$ is not linear in the driving flow $\boldsymbol{v}_{0}$, the resulting loads $\boldsymbol{F}_{2, c}$ and $\boldsymbol{T}_{2, c}$ generally lead to irreversible motion. Our focus is accordingly centred at the 'diffusio-osmotic' flow component $\boldsymbol{v}_{2, c}$. We define:

$$
\boldsymbol{v}_{2, c}=\alpha \mathscr{H}^{2}(\zeta) \tilde{\boldsymbol{v}}, \quad p_{2, c}=\alpha \mathscr{H}^{2}(\zeta) \tilde{p}
$$

The flow $(\tilde{\boldsymbol{v}}, \tilde{p})$ is governed by the homogeneous Stokes and continuity equations and the requirement of far-field decay; it is engendered by the slip condition

$$
\tilde{\boldsymbol{v}}=-\nabla_{s} \tilde{c} \quad \text { on } \quad s .
$$



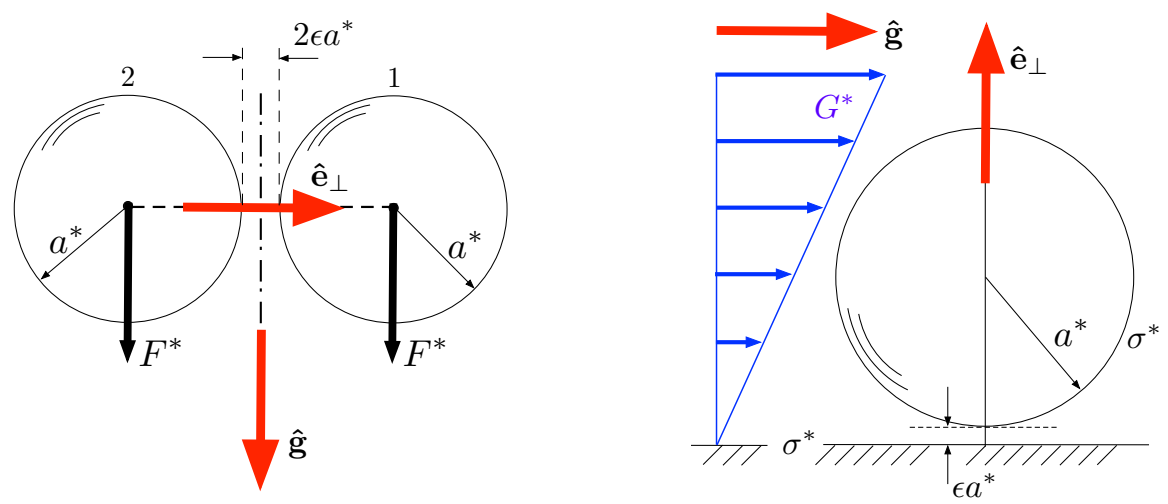

FiguRE 1. (a) Force-induced problem: Two identical spheres sedimenting perpendicularly to their line of centres under the action of an external force. (b) Flow-induced problem: A spherical particle suspended in proximity to a solid wall and exposed to a simple shear flow.

As the streaming potential does not result in any irreversibility, we do not bother calculating it. This is in marked difference with the limit of large Péclet numbers (Yariv et al. 2011), where irreversibility is induced by that potential through Maxwell stresses.

The preceding simplifications, allowing to limit the attention to the diffusio-osmotic flow component, follow from the assumed quasi-steadiness. In an inherently unsteady problem, the temporal-derivative term in (2.20) constitutes an additional source of irreversibility. An example of such a problem is the sedimentation of a particle towards a solid wall.

\section{Formulation of two problems}

We here demonstrate electroviscous irreversibility by considering in detail two problems which involve spherical particles (radius $a^{*}$ ) moving in an electrolyte solution. The first entails two identical torque-free particles settling in an otherwise quiescent unbounded fluid under an external force (gravity, buoyancy, etc.) acting perpendicular to their line of centres. The force magnitude is $F^{*}$ and its direction is specified by the unit vector $\hat{\boldsymbol{g}}$. The second problem we analyse is driven by an imposed flow; it entails a single particle suspended in proximity to a solid wall and exposed to simple shear flow (shearrate magnitude $G^{*}$, direction $\hat{\boldsymbol{g}}$ ). The two problems are described in figure 1 . Noting the mirror symmetry of the first problem about the mid-plane, both problems can be formulated using a configuration consisting of a plane, denoted hereafter as 'the' plane, and a single sphere (particle ' 1 ' in the sedimentation problem), denoted hereafter as 'the' particle. The geometry of both problems is described by a single fixed vector $\hat{\boldsymbol{e}}_{\perp}$ perpendicular to that plane, as shown in figure 1.

We employ the dimensionless notation of $\S 2$. In the first problem we employ $F^{*} / \mu^{*} a^{*}$ as the velocity scale $v^{*}$. The external force and torque on particle ' 1 ' are thus (see (2.33))

$$
\boldsymbol{F}_{e}=\hat{\boldsymbol{g}}, \quad \boldsymbol{T}_{e}=\mathbf{0} .
$$

In the second problem we choose the velocity scale $v^{*}$ as $a^{*} G^{*}$. Here the external force and torque vanish:

$$
\boldsymbol{F}_{e}=\mathbf{0}, \quad \boldsymbol{T}_{e}=\mathbf{0} .
$$




\subsection{Motion in the absence of electrokinetic effects}

Let $\boldsymbol{U}$ and $\boldsymbol{\Omega}$ respectively denote the rigid-body rectilinear and angular velocities of the particle in the absence of electrokinetic effects ( $\boldsymbol{U}$ is the instantaneous velocity of the particle centre). Simple symmetry arguments (Jeffrey 1996, see also (A 5)-(A 6)) show that the vectors $\boldsymbol{U}$ and $\boldsymbol{\Omega}$ are respectively aligned in the directions of $\hat{\boldsymbol{g}}$ and $\hat{\boldsymbol{e}}_{\perp} \times \hat{\boldsymbol{g}}$,

$$
\boldsymbol{U}=\mathscr{U} \hat{\boldsymbol{g}}, \quad \Omega=\Omega \hat{\boldsymbol{e}}_{\perp} \times \hat{\boldsymbol{g}} .
$$

The particle thus retains a constant separation distance, say $\epsilon$, from the plane. The velocities $\boldsymbol{U}$ and $\boldsymbol{\Omega}$ are obtained by balancing external and hydrodynamic loads. In the first problem, the latter loads consist of resistance to rigid-body motion (see appendix). In the second problem, there is an additional contribution owing to the ambient shear flow (with a unity shear-rate magnitude). Specifically, the force balance in the $\hat{\boldsymbol{g}}$-direction and the torque balance in the direction of $\hat{\boldsymbol{e}}_{\perp} \times \hat{\boldsymbol{g}}$ respectively yield (see (A 5)-(A 6))

$$
f=f_{\|} \mathscr{U}+m \Omega, \quad t=t_{\|} \Omega+m \mathscr{U},
$$

wherein the force $\left(f_{\|}\right)$, torque $\left(t_{\|}\right)$, and coupling $(m)$ coefficients, which are functions of $\epsilon$, are defined in the Appendix. In the first problem, $f=1$ and $t=0$ represent external force and torque coefficients. In the second problem, $f$ and $t$ represent the hydrodynamic loads on a stationary particle in a simple shear flow of unit shear rate. Just like the resistance coefficients, the latter are also functions of $\epsilon$.

Inversion of (4.4) yields $\mathscr{U}$ and $\Omega$ as functions of $\epsilon$ :

$$
\mathscr{U}(\epsilon)=\frac{f t_{\|}-m t}{t_{\|} f_{\|}-m^{2}}, \quad \Omega(\epsilon)=\frac{t f_{\|}-m f}{f_{\|} t_{\|}-m^{2}} .
$$

\subsection{The electroviscous problem}

We employ a Cartesian reference system, where the $x$-axis points in the external-force (or flow) direction $\hat{\boldsymbol{g}}$. The $z$-axis points in the direction of $\hat{\boldsymbol{e}}_{\perp}$ and passes through the centre of particle ' 1 .' The plane $z=0$ coincides with the symmetry plane in the first problem and the solid wall in the second: see figure 2 . To conform with the requirement of quasi-steady transport, as defined in $\S 3$, the system is instantaneously moving with velocity $\mathscr{U} \hat{\boldsymbol{e}}_{x}$.

In this reference frame, the driving flow $\left(\boldsymbol{v}_{0}, p_{0}\right)$ satisfies the continuity and Stokes equations together with the no-slip condition on the particle boundary,

$$
\boldsymbol{v}_{0}=\hat{\boldsymbol{e}}_{y} \Omega \times \hat{\boldsymbol{n}}
$$

In the first problem, $\boldsymbol{v}_{0}$ satisfies the symmetry conditions

$$
\frac{\partial u_{0}}{\partial z}=\frac{\partial v_{0}}{\partial z}=w_{0}=\frac{\partial p_{0}}{\partial z}=0
$$

at $z=0$, and the approach to a uniform stream,

$$
\boldsymbol{v}_{0} \rightarrow-\hat{\boldsymbol{e}}_{x} \mathscr{U},
$$

as $|\boldsymbol{x}| \rightarrow \infty(\boldsymbol{x}=(x, y, z)$ being the position vector $)$. In the second problem, it satisfies the no-slip condition

at $z=0$, and the condition

$$
\boldsymbol{v}_{0}=-\hat{\boldsymbol{e}}_{x} \mathscr{U}
$$

$$
\boldsymbol{v}_{0} \sim G \cdot \boldsymbol{x}-\hat{\boldsymbol{e}}_{x} \mathscr{U},
$$

where $G=\hat{\boldsymbol{e}}_{x} \hat{\boldsymbol{e}}_{z}$, as $|\boldsymbol{x}| \rightarrow \infty$. The problem is closed by imposing the conditions (2.34) 

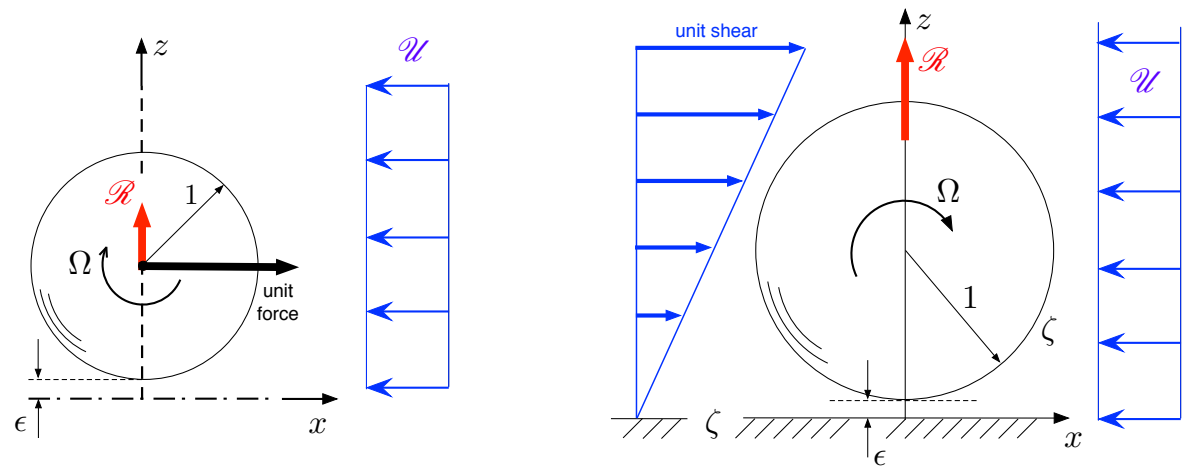

FIgURE 2. Dimensionless description: (a) particle-pair sedimentation; (b) particle in shear flow. The velocities $\mathscr{U}$ and $\Omega$ represent the relative rigid-body motion of the particle relative to the fluid in the absence of electrokinetic effects. Also shown is the electroviscous repulsive force, of magnitude $\mathscr{R}$.

of a freely suspended particle, which are satisfied by the very construction of $\mathscr{U}$ and $\Omega$. We shall consider $\boldsymbol{v}_{0}$, which is linear in $\mathscr{U}$ and $\Omega$, as known for any given value of $\epsilon$.

In what follows, we proceed to the calculation of the electrokinetic flow $\boldsymbol{v}_{2}$. Specifically, following the arguments of $\S 3$, we focus upon the calculation of the diffusio-osmotic subfield $\boldsymbol{v}_{2, c}$, the only component of (2.28) responsible for irreversible repulsion. We accordingly limit our attention to the problems governing the rescaled fields $\tilde{c}$ and $\tilde{\boldsymbol{v}}$, respectively defined by (3.2) and (3.6). In doing so, it is convenient to employ cylindrical coordinates $(r, \theta, z)$, where the azimuthal angle $\theta$ is measured counter-clockwise from the $x$-axis in the $x y$-plane. The radial, azimuthal, and axial components of $\boldsymbol{v}_{0}$ are denoted $\left(u_{0}, v_{0}, w_{0}\right)$. We employ a similar notation for the diffusio-osmotic flow $\tilde{\boldsymbol{v}}$, denoting the respective components $(\tilde{u}, \tilde{v}, \tilde{w})$.

The rescaled salt perturbation $\tilde{c}$ is governed by the advection-diffusion equation (cf. (3.3))

$$
\frac{\partial \tilde{c}}{\partial r^{2}}+\frac{1}{r} \frac{\partial \tilde{c}}{\partial r}+\frac{1}{r^{2}} \frac{\partial \tilde{c}}{\partial \theta^{2}}+\frac{\partial \tilde{c}}{\partial z^{2}}=P e\left(u_{0} \frac{\partial \tilde{c}}{\partial r}+\frac{v_{0}}{r} \frac{\partial \tilde{c}}{\partial \theta}+w_{0} \frac{\partial \tilde{c}}{\partial z}\right),
$$

the inhomogeneous boundary condition on the particle surface (3.4), and far-field decay. The pertinent boundary condition on the plane $z=0$ depends on the problem considered. In the first $\tilde{c}$ satisfies the symmetry condition

$$
\frac{\partial \tilde{c}}{\partial z}=0 \quad \text { at } \quad z=0,
$$

while in the second it satisfies the inhomogeneous Neumann condition (cf. (3.4))

$$
\frac{\partial \tilde{c}}{\partial z}=-\frac{\partial p_{0}}{\partial z} \quad \text { at } \quad z=0
$$

The flow $\tilde{\boldsymbol{v}}$, induced by the diffusio-osmotic slip condition (3.7), is governed by the continuity equation (see $(2.24 a)$ )

$$
\frac{1}{r} \frac{\partial}{\partial r}(r \tilde{u})+\frac{1}{r} \frac{\partial \tilde{v}}{\partial \theta}+\frac{\partial \tilde{w}}{\partial z}=0
$$


the homogenous Stokes equations (see $(2.24 b)$ )

$$
\begin{gathered}
\frac{\partial \tilde{p}}{\partial r}=\nabla^{2} \tilde{u}-\frac{\tilde{u}}{r^{2}}-\frac{2}{r^{2}} \frac{\partial \tilde{v}}{\partial \theta}, \\
\frac{1}{r} \frac{\partial \tilde{p}}{\partial \theta}=\nabla^{2} \tilde{v}+\frac{2}{r^{2}} \frac{\partial \tilde{u}}{\partial \theta}-\frac{\tilde{v}}{r^{2}}, \\
\frac{\partial \tilde{p}}{\partial z}=\nabla^{2} \tilde{w} ;
\end{gathered}
$$

and far-field decay. In the first problem $\tilde{\boldsymbol{v}}$ satisfies the symmetry conditions

$$
\frac{\partial \tilde{u}}{\partial z}=\frac{\partial \tilde{v}}{\partial z}=\tilde{w}=\frac{\partial \tilde{p}}{\partial z}=0
$$

at $z=0$; in the second problem it vanishes there.

Once the flow $(\tilde{\boldsymbol{v}}, \tilde{p})$ is determined we can calculate the repulsive force $\mathscr{R}=\hat{\boldsymbol{e}}_{z} \cdot \boldsymbol{F}_{2, c}$ acting on the particle, see figure 2. Using the rescaling (3.6), it is given by

$$
\mathscr{R}=\alpha \mathscr{H}^{2}(\zeta) \hat{\boldsymbol{e}}_{z} \cdot \oint \mathrm{d} A \hat{\boldsymbol{n}} \cdot \tilde{N}
$$

wherein (cf. (2.12))

$$
\tilde{N}=-\tilde{p} \boldsymbol{I}+(\boldsymbol{\nabla} \tilde{\boldsymbol{v}})+(\boldsymbol{\nabla} \tilde{\boldsymbol{v}})^{\dagger}
$$

is the Newtonian stress associated with $\tilde{\boldsymbol{v}}$.

For future reference, we note that the symmetry of the driving flow problem about the $x z$-plane allows to write the components $\left(u_{0}, v_{0}, w_{0}\right)$ of $\boldsymbol{v}_{0}$ and the pressure $p_{0}$ as (O'Neill 1969)

$$
\bar{u}(r, z) \cos \theta, \quad \bar{v}(r, z) \sin \theta, \quad \bar{w}(r, z) \cos \theta, \quad \bar{p}(r, z) \cos \theta .
$$

It is important to note that, due to the presence of the advection term in (3.3), neither $\tilde{c}$ nor $(\tilde{\boldsymbol{v}}, \tilde{p})$ can in general be represented in a similar manner.

\section{Near-contact limit: preliminaries}

We focus hereafter upon the near-contact limit $\epsilon \ll 1$ (but still $\delta \ll \epsilon$ ). This limit is interesting because, as will become evident, it results in a relatively large repulsion. Consideration of this limit also allows us to take advantage of known results which apply in the absence of electrokinetic effects. In particular, the flow $\boldsymbol{v}_{0}$, along with the associated resistance coefficients, has been calculated in the near-contact limit by O'Neill and coworkers for the two configurations we consider in the present contribution. These calculations were carried out using inner-outer matched asymptotic expansions. The outer region corresponds to the particle scale, where at leading order the particle appears to touch the plane $z=0$. The inner region constitutes the narrow gap between the particle and the plane $z=0$, and is described by the strained coordinates

$$
Z=z / \epsilon, \quad R=r / \epsilon^{1 / 2} .
$$

In these coordinates, the particle boundary is $Z=H(R ; \epsilon)$, where $H$ is provided by the expansion

$$
H(R ; \epsilon)=H^{(0)}(R)+\epsilon H^{(1)}(R)+\cdots ;
$$

in particular (see O'Neill \& Stewartson 1967),

$$
H^{(0)}(R)=1+\frac{R^{2}}{2} .
$$


As will become evident, the final results are independent of $H^{(1)}$; since no confusion should arise, we omit hereafter the superscript from $H^{(0)}$.

\subsection{The driving flow $\boldsymbol{v}_{0}$ and the associated resistance coefficients}

The calculation of $\boldsymbol{v}_{0}$ in the first problem, of two settling spheres, was carried out by O'Neill (1969) in a laboratory-fixed reference frame, where the fluid at infinity is at rest. In what follows, we present his results in our co-moving coordinates. The linearity of the governing equations allows to decompose the problem into two parts: (i) two stationary particles in a uniform stream $-\mathscr{U} \hat{\boldsymbol{e}}_{x}$ perpendicular to their line of centres; and (ii) two particles counter-rotating with velocities $\pm \Omega \hat{\boldsymbol{e}}_{y}$ without translation. These two parts differ significantly: the former is described at leading order by simply setting $\epsilon=0$ (i.e. two spheres touching), and does not really require the use of matched asymptotic expansions; the latter is singular in $\epsilon$ (due to the intense shear rate within the narrow gap) and is characterised by an $O\left(\epsilon^{-3 / 2}\right)$ large pressure within the inner gap region.

The appropriate gap-region scalings of the velocity amplitudes are

$$
\bar{u}=\bar{U}(R, Z ; \epsilon), \quad \bar{v}=\bar{V}(R, Z ; \epsilon), \quad \bar{w}=\epsilon^{1 / 2} \bar{W}(R, Z ; \epsilon),
$$

while the pressure there is

$$
\bar{p}=\epsilon^{-3 / 2} \bar{P}(R, Z ; \epsilon) .
$$

At leading order, these fields are affected only by the 'singular' pure-rotation component of the problem. In particular, employing the generic expansion

$$
\bar{F}(R, Z ; \epsilon)=\bar{F}^{(0)}(R, Z)+\epsilon \bar{F}^{(1)}(R, Z)+\cdots,
$$

the results of O'Neill (1969) read

$$
\bar{U}^{(0)}=\frac{1}{2}\left(Z^{2}-H^{2}(R)\right) \frac{d \bar{P}^{(0)}}{d R}-\Omega, \quad \bar{V}^{(0)}=\frac{1}{2}\left(H^{2}(R)-Z^{2}\right) \frac{\bar{P}^{(0)}}{R}+\Omega,
$$

where

$$
\bar{P}^{(0)}=\frac{3 R}{5 H^{2}(R)} \Omega
$$

is a function of $R$ alone. As will become evident, we do not require here the comparable explicit description in the outer region.

The solution of O'Neill (1969) also provides the asymptotic expansions of the resistance coefficients appearing in (4.4). Thus, in the limit $\epsilon \rightarrow 0$ O'Neill (1969) obtained (with an error smaller than some power of $\epsilon$ )

$$
f_{\|} / 6 \pi \approx 0.72426, \quad m / 8 \pi \approx 0.11843,
$$

where the two $O(1)$ terms are contributed by the outer region, and

$$
t_{\|} / 8 \pi \approx-\frac{3}{20} \ln \epsilon+0.62664
$$

where the terms are contributed by both the inner and outer regions. The logarithmic divergence of $t_{\|}$is associated with the large pressure associated with the pure-rotation problem.

In the second problem it is convenient to decompose the driving flow into three components respectively representing (i) a stationary sphere suspended near a wall, with the fluid-wall environment moving with velocity $-\hat{\boldsymbol{e}}_{x} \mathscr{U}$; (ii) a sphere rotating with a velocity $\hat{\boldsymbol{e}}_{y} \Omega$ relative to an otherwise quiescent fluid and a stationary wall; and (iii) stationary sphere and wall exposed to the imposed shear flow $G \cdot \boldsymbol{x}$. In the near-contact limit, 
these sub-problems were respectively analysed by O'Neill \& Stewartson (1967), Cooley \& O'Neill (1968), and O'Neill (1968). There is here a fundamental difference between the first two sub-problems, where the limit $\epsilon \rightarrow 0$ is a singular one, associated with $O\left(\epsilon^{-3 / 2}\right)$ large gap pressures, and the third one, wherein no relative motion is imposed between the sphere and the wall and the limit $\epsilon \rightarrow 0$ is regular. The inner scalings (5.4)-(5.5) are accordingly applicable, now with the pertinent fields affected only by the ('singular') components (i) and (ii) of the flow. (Remarkably, then, while the second problem is forced by the imposed shear, the direct contribution of flow component (iii) in that problem does not affect the subsequent calculation.) In particular, the translation component yields here (O’Neill \& Stewartson 1967)

$$
\bar{U}^{(0)}=\frac{Z(Z-H)}{2} \frac{\mathrm{d} P^{(0)}}{\mathrm{d} R}+\mathscr{U}(Z-1), \quad \bar{V}^{(0)}=-\frac{Z(Z-H)}{2} \frac{P^{(0)}}{R}-\mathscr{U}(Z-1),(5.11 a, b)
$$

wherein

$$
\bar{P}^{(0)}=\frac{6 R}{5 H^{2}(R)} \mathscr{U},
$$

while the rotation component gives (Cooley \& O’Neill 1968)

$$
\bar{U}^{(0)}=\frac{Z(Z-H)}{2} \frac{\mathrm{d} P^{(0)}}{\mathrm{d} R}-\Omega Z, \quad \bar{V}^{(0)}=-\frac{Z(Z-H)}{2} \frac{P^{(0)}}{R}+\Omega Z,
$$

wherein

$$
\bar{P}^{(0)}=\frac{6 R}{5 H^{2}(R)} \Omega
$$

The calculations of O'Neill \& Stewartson (1967) provide the force and coupling coefficients (see Appendix) for a particle translating near a wall, namely

$$
f_{\|} / 6 \pi \approx \frac{8}{15} \ln \frac{2}{\epsilon}+0.58461, \quad m / 8 \pi \approx-\frac{1}{10} \ln \frac{2}{\epsilon}+0.26227 .
$$

The comparable torque coefficient,

$$
t_{\|} / 8 \pi \approx-\frac{2}{5} \ln \epsilon+0.37085
$$

is provided by Cooley \& O'Neill (1968). The terms in (5.15)-(5.16) are contributed by both the inner and outer regions. The force and torque in sub-problem (iii), $f \hat{\boldsymbol{e}}_{x}$ and $t \hat{\boldsymbol{e}}_{y}$, are contributed at leading order by the outer region (O'Neill 1968):

$$
f / 6 \pi \approx 1.7009, \quad t / 8 \pi \approx 0.471996 .
$$

In approximations (5.9)-(5.10) and (5.15)-(5.17) the error is asymptotically smaller than some positive power of $\epsilon$. Substituting of (5.9)-(5.10) into (4.5) provides the rigidbody velocities $\mathscr{U}$ and $\Omega$ in the settling problem. In the shear problem these velocities are obtained using (5.15)-(5.17). As is the common practice in asymptotic analyses with singular algebraic terms, logarithmic terms are considered as effectively belonging to $O(1)$ (Hinch 1991). With this interpretation we consider $\mathscr{U}$ and $\Omega$, as provided by (4.5), to be a (known) $O(1)$ numbers.

In the present context, the $O\left(\epsilon^{-3 / 2}\right)$ pressure scaling associated with the singular components of the flow (counter-rotation in the first problem, counter rotation and translation in the second problem) is expected (see (3.4)) to result in a comparable scaling of the associated salt perturbation. Moreover, because of the structure of the electroviscous problem, this is anticipated to result here in an algebraic singularity of the repulsive force as $\epsilon \rightarrow 0$. As our goal is to calculate the repulsion to leading order in $\epsilon$, this suggests that the salt perturbation needs to be calculated only within the narrow gap region. 
Before proceeding further it is worthwhile to note the following points for future reference. First, use of (5.3) implies that, in the inner region, the vector

$$
-\hat{\boldsymbol{e}}_{z}+\hat{\boldsymbol{e}}_{r} \epsilon^{1 / 2} R[1+O(\epsilon)]
$$

is normal to the particle surface. Substitution into (2.27) yields the corresponding expansion of the surface-gradient operator

$$
\begin{aligned}
\nabla_{s}=\hat{\boldsymbol{e}}_{r} \epsilon^{-1 / 2}\left[\frac{\partial}{\partial R}+R \frac{\partial}{\partial Z}+\right. & O(\epsilon)] \\
& +\hat{\boldsymbol{e}}_{\theta} \epsilon^{-1 / 2} \frac{1}{R} \frac{\partial}{\partial \theta}+\hat{\boldsymbol{e}}_{z}\left[R \frac{\partial}{\partial R}+R^{2} \frac{\partial}{\partial Z}+O(\epsilon)\right]
\end{aligned}
$$

Second, as the Stokes-flow pressure field $p_{0}$ must be harmonic, $\bar{P}^{(1)}$ is related to $\bar{P}^{(0)}$ via the differential equation

$$
\frac{\partial^{2} \bar{P}^{(1)}}{\partial Z^{2}}+\left(\frac{\mathrm{d}^{2}}{\mathrm{~d} R^{2}}+\frac{1}{R} \frac{\mathrm{d}}{\mathrm{d} R}-\frac{1}{R^{2}}\right) \bar{P}^{(0)}=0
$$

\section{Salt transport}

In light of the boundary condition (3.4) and the pressure scaling (5.5) we define

$$
\tilde{c}=\epsilon^{-3 / 2} C(R, Z, \theta ; \epsilon) .
$$

The rescaled concentration $C$ is governed by the advection-diffusion equation (cf. (4.11))

$$
\begin{aligned}
\frac{\partial^{2} C}{\partial Z^{2}}+\epsilon\left(\frac{\partial^{2}}{\partial R^{2}}+\frac{1}{R} \frac{\partial}{\partial R}+\right. & \left.\frac{1}{R^{2}} \frac{\partial^{2}}{\partial \theta^{2}}\right) C \\
& =P e \epsilon^{3 / 2}\left(\bar{U} \cos \theta \frac{\partial}{\partial R}+\bar{V} \frac{\sin \theta}{R} \frac{\partial}{\partial \theta}+\bar{W} \cos \theta \frac{\partial}{\partial Z}\right) C,
\end{aligned}
$$

together with the salt-flux condition (3.4), which, upon using (5.18), reads

$$
\frac{\partial C}{\partial Z}-\epsilon(R+\cdots) \frac{\partial C}{\partial R}=-\left[\frac{\partial \bar{P}}{\partial Z}-\epsilon(R+\cdots) \frac{\partial \bar{P}}{\partial R}\right] \cos \theta \quad \text { at } \quad Z=H(R ; \epsilon) .
$$

In the first problem, $C$ additionally satisfies the symmetry condition at $Z=0, \partial C / \partial Z=$ 0 . In the second problem, $C$ satisfies there the salt-flux condition (cf. (3.4)):

$$
\frac{\partial C}{\partial Z}=-\frac{\partial \bar{P}}{\partial Z} \cos \theta
$$

Given (4.18), however, $\partial P / \partial Z$ vanishes in the first problem at $Z=0$; it follows that (6.4) applies for both problems. Last, asymptotic matching of (6.1) with the $O(1)$ concentration outside the gap implies:

$$
\lim _{R \rightarrow \infty} C=0 .
$$

Equations (6.2)-(6.3) suggest the expansion

$$
\begin{aligned}
C(R, Z, \theta ; \epsilon)=C^{(0)}(R, Z, \theta)+\epsilon^{1 / 2} C^{(1 / 2)}(R, Z, \theta) & \\
& +\epsilon C^{(1)}(R, Z, \theta)+\epsilon^{3 / 2} C^{(3 / 2)}(R, Z, \theta)+\cdots
\end{aligned}
$$


Substituting (6.6) into (6.2) yields at the leading four asymptotic orders:

$$
\begin{gathered}
\frac{\partial^{2} C^{(0)}}{\partial Z^{2}}=0 \\
\frac{\partial^{2} C^{(1 / 2)}}{\partial Z^{2}}=0, \\
\frac{\partial^{2} C^{(1)}}{\partial Z^{2}}+\left(\frac{\partial^{2}}{\partial R^{2}}+\frac{1}{R} \frac{\partial}{\partial R}+\frac{1}{R^{2}} \frac{\partial^{2}}{\partial \theta^{2}}\right) C^{(0)}=0,
\end{gathered}
$$

and

$$
\begin{aligned}
\frac{\partial^{2} C^{(3 / 2)}}{\partial Z^{2}}+\left(\frac{\partial^{2}}{\partial R^{2}}\right. & \left.+\frac{1}{R} \frac{\partial}{\partial R}+\frac{1}{R^{2}} \frac{\partial^{2}}{\partial \theta^{2}}\right) C^{(1 / 2)} \\
& =\operatorname{Pe}\left(\bar{U}^{(0)} \cos \theta \frac{\partial}{\partial R}+\bar{V}^{(0)} \frac{\sin \theta}{R} \frac{\partial}{\partial \theta}+\bar{W}^{(0)} \cos \theta \frac{\partial}{\partial Z}\right) C^{(0)}
\end{aligned}
$$

Since $\bar{P}^{(0)}$ is independent of $Z$, the corresponding conditions at $Z=0$ are

$$
\frac{\partial C^{(n / 2)}}{\partial Z}=0 \quad \text { for } \quad n=0,1,3
$$

and

$$
\frac{\partial C^{(1)}}{\partial Z}=-\frac{\partial \bar{P}^{(1)}}{\partial Z} \cos \theta
$$

Making use of the salt-flux condition (6.3) and noting that $\bar{P}^{(0)}$ is a function of $R$ alone we obtain at $Z=H(R)$

$$
\begin{gathered}
\frac{\partial C^{(0)}}{\partial Z}=0, \\
\frac{\partial C^{(1 / 2)}}{\partial Z}=0, \\
\frac{\partial C^{(1)}}{\partial Z}+\left(H^{(1)} \frac{\partial^{2}}{\partial Z^{2}}-R \frac{\partial}{\partial R}\right) C^{(0)}=-\left(\frac{\partial \bar{P}^{(1)}}{\partial Z}-R \frac{\mathrm{d} \bar{P}^{(0)}}{\mathrm{d} R}\right) \cos \theta,
\end{gathered}
$$

and

$$
\frac{\partial C^{(3 / 2)}}{\partial Z}+\left(H^{(1)} \frac{\partial^{2}}{\partial Z^{2}}-R \frac{\partial}{\partial R}\right) C^{(1 / 2)}=0
$$

Equations $(6.7 a),(6.8 a)$ and $(6.9 a)$ imply that $C^{(0)}$ is independent of $Z$ and is accordingly a function of $R$ and $\theta$ alone. Its explicit form is found using the solvability condition governing $C^{(1)}$, obtained as follows. Integration of $(6.7 c)$ from $Z=0$ to $Z=H$ in conjunction with $(6.8 b)$ yields

$$
\left.\frac{\partial C^{(1)}}{\partial Z}\right|_{Z=H}+\left.\frac{\partial \bar{P}_{1}}{\partial Z}\right|_{Z=0} \cos \theta=-H\left(\frac{\partial^{2}}{\partial R^{2}}+\frac{1}{R} \frac{\partial}{\partial R}+\frac{1}{R^{2}} \frac{\partial^{2}}{\partial \theta^{2}}\right) C^{(0)} .
$$

Also, integration of (5.20) across the gap gives

$$
\left.\frac{\partial \bar{P}^{(1)}}{\partial Z}\right|_{Z=H}=\left.\frac{\partial \bar{P}^{(1)}}{\partial Z}\right|_{Z=0}-H\left(\frac{\mathrm{d}^{2}}{\mathrm{~d} R^{2}}+\frac{1}{R} \frac{\mathrm{d}}{\mathrm{d} R}-\frac{1}{R^{2}}\right) \bar{P}^{(0)} .
$$


Substitution of $(6.10)-(6.11)$ into $(6.9 c)$ yields:

$$
\begin{aligned}
\left\{\frac{\partial^{2}}{\partial R^{2}}+\left(\frac{1}{R}+\frac{R}{H(R)}\right) \frac{\partial}{\partial R}\right. & \left.+\frac{1}{R^{2}} \frac{\partial^{2}}{\partial \theta^{2}}\right\} C^{(0)} \\
& =-\cos \theta\left\{\frac{\mathrm{d}^{2}}{\mathrm{~d} R^{2}}+\left(\frac{1}{R}+\frac{R}{H(R)}\right) \frac{\mathrm{d}}{\mathrm{d} R}-\frac{1}{R^{2}}\right\} \bar{P}^{(0)}
\end{aligned}
$$

The solution of this equation which also satisfies (6.5) is clearly

$$
C^{(0)}=-\bar{P}^{(0)}(R) \cos \theta .
$$

This result was to be expected: As advection only affects the transport equation (6.2) at $O\left(\epsilon^{3 / 2}\right)$, the problem governing $C^{(0)}$ and the solvability condition obtained from the problem governing $C^{(1)}$ are clearly unaffected by it. Going back to the original problem governing $\tilde{c}$, we see that in the absence of advection this field is harmonic, whence condition (3.4) implies a simple proportionality to the harmonic field $p_{0}$.

Given the dependence of $C^{(0)}$ upon $\theta$, the corresponding slip (3.7) is anti-symmetric, and so does not contribute to the repulsive force. Thus, we need to go to a higher asymptotic order and evaluate $C^{(1 / 2)}$. From $(6.7 b),(6.8 a)$ and $(6.9 b)$ we find that $C^{(1 / 2)}$, just like $C^{(0)}$, is a function of $R$ and $\theta$ alone. The differential equation governing $C^{(1 / 2)}$ is obtained from the solvability condition governing $C^{(3 / 2)}$; thus, integrating $(6.7 d)$ over $0<Z<H(R)$ and substituting $(6.8 a)$ and $(6.9 d)$ yields

$$
\begin{aligned}
& \left\{\frac{\partial^{2}}{\partial R^{2}}+\left(\frac{1}{R}+\frac{R}{H(R)}\right) \frac{\partial}{\partial R}+\frac{1}{R^{2}} \frac{\partial^{2}}{\partial \theta^{2}}\right\} C^{(1 / 2)} \\
& =\frac{P e}{2 H(R)}\left\{(1-\cos 2 \theta) \frac{\bar{P}^{(0)}}{R} \int_{0}^{H} \bar{V}^{(0)} \mathrm{d} Z-(1+\cos 2 \theta) \frac{\mathrm{d} \bar{P}^{(0)}}{\mathrm{d} R} \int_{0}^{H} \bar{U}^{(0)} \mathrm{d} Z\right\} .
\end{aligned}
$$

Clearly, $C^{(1 / 2)}(R, \theta)$ can be decomposed into two terms, one linear in $\cos 2 \theta$ and the other, say $\bar{C}^{(1 / 2)}(R)$, independent of $\theta$. Since the former, again, does not contribute to the repulsive force, we focus on the contribution of the latter, governed by the following ordinary differential equation

$$
\begin{aligned}
\left\{\frac{\mathrm{d}^{2}}{\mathrm{~d} R^{2}}+\left(\frac{1}{R}+\frac{R}{H(R)}\right)\right. & \left.\frac{\mathrm{d}}{\mathrm{d} R}\right\} \bar{C}^{(1 / 2)} \\
& =\frac{P e}{2 H(R)}\left\{\frac{\bar{P}^{(0)}}{R} \int_{0}^{H} \bar{V}^{(0)} \mathrm{d} Z-\frac{\mathrm{d} \bar{P}^{(0)}}{\mathrm{d} R} \int_{0}^{H} \bar{U}^{(0)} \mathrm{d} Z\right\}
\end{aligned}
$$

As will become evident, calculation of the repulsive force engendered by $\bar{C}^{(1 / 2)}$ does not require the explicit solution of this equation.

\section{Diffusio-osmotic flow}

By the linearity of the Stokes equations and the slip condition (3.7) we can solve independently for the axisymmetric part of $\tilde{\boldsymbol{v}}$ induced by the axisymmetric part $\bar{C}^{(1 / 2)}(R)$ of $C^{(1 / 2)}$. Since the leading-order term in (6.6) does not contribute to the repulsive force, equations (3.7) and (6.1) suggest writing the radial and axial components of the axisymmetric part of $\tilde{\boldsymbol{v}}$ as

$$
\epsilon^{-3 / 2} U^{(0)}(R, Z)+\cdots, \quad \epsilon^{-1} W^{(0)}(R, Z)+\cdots,
$$


with the corresponding pressure being

$$
\epsilon^{-3} P^{(0)}(R, Z)+\cdots
$$

In what follows, it proves useful to treat the two problems separately.

\subsection{Settling problem}

Using (5.19), the slip condition (3.7) reads at leading order

$$
U^{(0)}=-\frac{\mathrm{d} \bar{C}^{(1 / 2)}}{\mathrm{d} R}, \quad W^{(0)}=-R \frac{\mathrm{d} \bar{C}^{(1 / 2)}}{\mathrm{d} R} \quad \text { at } \quad Z=H(R) .
$$

The symmetry conditions (4.18) yield

$$
\frac{\partial U^{(0)}}{\partial Z}=0, \quad W^{(0)}=0 \quad \text { at } \quad Z=0 .
$$

With definitions (7.1)-(7.2), the leading-order balances of the radial and axial momentum equations (4.15) and (4.17) are

$$
\frac{\partial P^{(0)}}{\partial R}=\frac{\partial^{2} U^{(0)}}{\partial Z^{2}}, \quad \frac{\partial P^{(0)}}{\partial Z}=0
$$

the latter implying that $P^{(0)}$ is a function of $R$ alone. The leading-order balance of the continuity equation $(4.14)$ is

$$
\frac{1}{R} \frac{\partial}{\partial R}\left(R U^{(0)}\right)+\frac{\partial W^{(0)}}{\partial Z}=0
$$

These equations are supplemented by the matching condition

$$
\lim _{R \rightarrow \infty} P^{(0)}=0
$$

The preceding problem is handled using the standard lubrication-flow procedure. Integration of the radial momentum equation (7.5a) in conjunction with $(7.3 a)$ and $(7.4 a)$ yields

$$
U^{(0)}=\frac{Z^{2}-H^{2}(R)}{2} \frac{\mathrm{d} P^{(0)}}{\mathrm{d} R}-\frac{\mathrm{d} \bar{C}^{(1 / 2)}}{\mathrm{d} R} .
$$

Substitution into (7.6), followed by integration over $Z$ in conjunction with $(7.3 b)-(7.4 b)$ yields an ordinary differential equation governing $P^{(0)}$ :

$$
\begin{aligned}
\left\{\frac{\mathrm{d}^{2}}{\mathrm{~d} R^{2}}+\left(\frac{1}{R}+\frac{3 R}{H(R)}\right) \frac{\mathrm{d}}{\mathrm{d} R}\right\} & P^{(0)} \\
& =-\frac{3}{H^{2}(R)}\left\{\frac{\mathrm{d}^{2}}{\mathrm{~d} R^{2}}+\left(\frac{1}{R}+\frac{R}{H(R)}\right) \frac{\mathrm{d}}{\mathrm{d} R}\right\} \bar{C}^{(1 / 2)} .
\end{aligned}
$$

Conveniently, the differential operator appearing in the right-hand side of (7.9) is the same as that governing $\bar{C}^{(1 / 2)}$ in (6.15). (This is why we did not bother calculating $\bar{C}^{(1 / 2)}$.) Thus, substitution of (6.15) provides the ordinary differential equation governing $P^{(0)}$

wherein

$$
\left\{\frac{\mathrm{d}^{2}}{\mathrm{~d} R^{2}}+\left(\frac{1}{R}+\frac{3 R}{H(R)}\right) \frac{\mathrm{d}}{\mathrm{d} R}\right\} P^{(0)}=\frac{\Lambda(R)}{R H^{3}(R)},
$$

$$
\Lambda(R)=\frac{3}{2} \operatorname{Pe} R\left\{\frac{\mathrm{d} \bar{P}^{(0)}}{\mathrm{d} R} \int_{0}^{H} \bar{U}^{(0)} \mathrm{d} Z-\frac{\bar{P}^{(0)}}{R} \int_{0}^{H} \bar{V}^{(0)} \mathrm{d} Z\right\} .
$$


This second-order equation can be written as a first-order equation governing $\mathrm{d} P^{(0)} / \mathrm{d} R$,

$$
\frac{\mathrm{d}}{\mathrm{d} R}\left\{R H^{3}(R) \frac{\mathrm{d} \bar{P}^{(0)}}{\mathrm{d} R}\right\}=\Lambda(R),
$$

whose solution is

$$
\frac{\mathrm{d} \bar{P}^{(0)}}{\mathrm{d} R}=\frac{1}{R H^{3}(R)}\left\{\text { constant }+\int_{0}^{R} \Lambda\left(R^{\prime}\right) \mathrm{d} R^{\prime}\right\} .
$$

It is readily verified that the particular integral is bounded as $R \rightarrow 0$; to ensure regularity of the pressure there we accordingly set the constant to zero. A subsequent integration in conjunction with the matching condition (7.7) yields

$$
P^{(0)}(R)=-\int_{R}^{\infty} \frac{\mathrm{d} R^{\prime}}{R^{\prime} H^{3}\left(R^{\prime}\right)} \int_{0}^{R^{\prime}} \mathrm{d} R^{\prime \prime} \Lambda\left(R^{\prime \prime}\right) .
$$

\subsection{Shear problem}

The scaling of the diffusio-osmotic flow driven by axisymmetric salt concentration $\bar{C}^{(1 / 2)}(R)$ is provided by (7.1)-(7.2). The flow is governed by (7.3)-(7.7), except that the symmetry conditions (7.4) at $Z=0$ are replaced by the slip and impermeability conditions

$$
U^{(0)}=-\frac{\mathrm{d} \bar{C}^{(1 / 2)}}{\mathrm{d} R}, \quad W^{(0)}=0 .
$$

Because of that difference, (7.8) changes to

$$
U^{(0)}=\frac{(Z-H(R))^{2}}{2} \frac{\mathrm{d} P^{(0)}}{\mathrm{d} R}-\frac{\mathrm{d} \bar{C}^{(1 / 2)}}{\mathrm{d} R} .
$$

Proceeding as before we obtain the following differential equation,

$$
\begin{aligned}
\left\{\frac{\mathrm{d}^{2}}{\mathrm{~d} R^{2}}+\left(\frac{1}{R}+\frac{3 R}{H(R)}\right) \frac{\mathrm{d}}{\mathrm{d} R}\right\} & P^{(0)} \\
& =-\frac{12}{H^{2}(R)}\left\{\frac{\mathrm{d}^{2}}{\mathrm{~d} R^{2}}+\left(\frac{1}{R}+\frac{R}{H(R)}\right) \frac{\mathrm{d}}{\mathrm{d} R}\right\} \bar{C}^{(1 / 2)},
\end{aligned}
$$

governing the diffusio-osmotic pressure.

\section{Repulsive force}

We can now calculate, to leading order in $\epsilon$, the repulsive irreversible force $\mathscr{R}=\hat{\boldsymbol{e}}_{z} \cdot \boldsymbol{F}_{2, c}$ acting on the particle. Due to the salt-perturbation scaling in the gap, this force is dominated by the gap-region stresses. In the inner region $\hat{\boldsymbol{n}} \sim-\hat{\boldsymbol{e}}_{z}$ thus the normal stress is dominated by the fluid pressure, see (4.20). Substitution of (7.2) into (4.19) followed by integration over the entire inner region yields

$$
\mathscr{R} \sim 2 \pi \alpha \mathscr{H}^{2}(\zeta) \epsilon^{-2} \int_{0}^{\infty} \mathrm{d} R R P^{(0)}(R) .
$$

In calculating the integral appearing in (8.1) we first consider the settling problem. Substituting (7.14) and interchanging the order of the two outermost integrations yields

$$
-\frac{1}{2} \int_{0}^{\infty} \frac{\mathrm{d} R R}{H^{3}(R)} \int_{0}^{R} \mathrm{~d} R^{\prime} \Lambda\left(R^{\prime}\right) .
$$


A subsequent interchange in the order of integration yields

$$
-\frac{1}{2} \int_{0}^{\infty} \mathrm{d} R^{\prime} \Lambda\left(R^{\prime}\right) \int_{R^{\prime}}^{\infty} \frac{\mathrm{d} R R}{H^{3}(R)} .
$$

Evaluating the inner integral finally gives

$$
-\frac{1}{4} \int_{0}^{\infty} \frac{\Lambda(R)}{H^{2}(R)} \mathrm{d} R .
$$

We conclude that

$$
\mathscr{R} \sim-\frac{1}{2} \pi \alpha \mathscr{H}^{2}(\zeta) \epsilon^{-2} \int_{0}^{\infty} \frac{\Lambda(R)}{H^{2}(R)} \mathrm{d} R .
$$

The function $\Lambda(R)$, defined in (7.11), is obtained via substitution of (5.7)-(5.8). Performing the quadrature in (8.5) eventually yields

$$
\mathscr{R}=\frac{6 \pi}{25} \epsilon^{-2} \alpha \mathscr{H}^{2}(\zeta) P e \Omega^{2}
$$

Recall that $\Omega$ is an $O(1)$ function of $\epsilon$, provided by (4.5b) and (5.9)-(5.10) with $f=1$ and $t=0$.

Considering now the shear problem we note that differential equation (7.17) governing $\bar{P}^{(0)}$ is the same as equation (7.9), obtained in the sedimentation problem, except for a factor 4 difference in the right-hand side. Since the diffusio-osmotic pressure is otherwise governed by the same homogeneous condition, namely (7.7), it follows that, upon accounting for that factor, expression (8.5) for the repulsion force between two sedimenting particles may be readily applied, giving:

$$
\mathscr{R} \sim-2 \pi \alpha \mathscr{H}^{2}(\zeta) \epsilon^{-2} \int_{0}^{\infty} \frac{\Lambda(R)}{H^{2}(R)} \mathrm{d} R .
$$

The function $\Lambda(R)$ is still given by (7.11), but the driving-flow variables appearing therein are now provided by the superposition of (5.11) (using (5.12)) and (5.13) (using (5.14)). Calculating the quadrature in (8.7) then furnishes the desired approximation:

$$
\mathscr{R}=\frac{24 \pi}{25} \epsilon^{-2} \alpha \mathscr{H}^{2}(\zeta) \operatorname{Pe}(\mathscr{U}+\Omega)^{2},
$$

wherein $\mathscr{U}$ and $\Omega$ are provided by (4.5) and (5.15)-(5.17) as $O(1)$ functions of $\epsilon$.

The resulting particle velocity (namely the rigid-body motion associated with $\boldsymbol{u}_{2}$ ) may by calculated using (2.35). In principle, this requires the calculation of all the electroviscous loads. It is however evident from the problem symmetry (see indeed (A $5 a$ )) that the calculation of the 'repulsive' velocity in the $z$-direction requires only the lateral repulsion calculated above. This velocity is thus given by $\mathscr{R} / f_{\perp}$. For small $\epsilon$, it is well known that $f_{\perp} \sim 6 \pi / \epsilon$, where the asymptotic error is smaller than some positive power of $\epsilon$ (Cox \& Brenner 1967). Thus, for $P e=O(1)$ the magnitude of the repulsive velocity is $O\left(\delta^{2} / \epsilon\right)$ relative to the driving flow. It is important to note however that experiments devised to detect electroviscous repulsion are typically based upon observing equilibrium positions which are determined by the balance with external forces (Alexander \& Prieve 1987). The quantity of interest in the present analysis is therefore the repulsive force.

\section{Discussion}

In parts 1 and 2 of this sequence we derived effective macroscale models enabling the calculation of electroviscous forces over the entire range of Péclet numbers. An important 
point revealed by these analyses is that, for both moderate and large Péclet numbers, the ratio of the induced-to-driving velocities scales as the square of the dimensionless Debye length $\delta$. These models show that apparently small nonlinear mechanisms can nevertheless generate Stokes-flow irreversibility, and hence their consequences may be important. For example, in the shear-induced lift experiments of Alexander \& Prieve (1987) and Bike, Lazarro \& Prieve (1995), electroviscous forces give rise to an otherwise impossible motion in a direction perpendicular to the driving motion.

The present paper illustrates irreversibility at moderate Péclet numbers by considering two prototypic problems. The first is side-by-side sedimentation of two spherical particles, and the second involves a spherical particle which is suspended in the vicinity of a planar solid wall and is exposed to a simple shear. Due the the reversibility properties of the Stokes equations, no lateral repulsion would be predicted in these configurations in the absence of electrokinetic effects. Our analyses are based on the macroscale model developed in part 2 of this sequence. In this limit, leading-order electroviscous forces are contributed by the Newtonian stresses accompanying the electrokinetic flow, which is driven by a combination of electro-osmosis and diffusio-osmosis. As the salt concentration is governed by an advection-diffusion equation, the latter mechanism is nonlinear in the driving flow and accordingly results in irreversible motion of suspended particles.

Since the effective boundary condition governing the salt-concentration distribution involves the pressure gradients of the driving flow, this repulsion becomes singular at near-contact configurations. In that limit, which is actually representative of realistic configurations, repulsion is dominated by the large 'diffusio-osmotic' pressure in the narrow-gap region. Use of appropriate lubrication approximations thus allows for an asymptotic treatment of the problem.

It turns out that the source of irreversibility is similar in both problems. The saltconcentration polarisation engendered by the advection of the driving flow possesses an axisymmetric component. The leading-order repulsion is contributed by the pressure distribution associated with the accompanying diffusio-osmotic flow. In calculating this repulsion, no need arises to assume small Péclet numbers; in fact, repulsion would not be detected if one were to use a small-Péclet-number linearisation. Remarkably, the ordinary differential equations which represent the advection-diffusion problem in the lubrication approximation do not need to be explicitly solved in order to obtain closedform approximations for the lateral repulsion.

It is convenient to express these approximations in a dimensional form, with $h^{*}$ denoting the small thickness $\epsilon a^{*}$. For the problem of side-by-side sedimentation under a force field of magnitude $F^{*}$ we have obtained the formula (cf. (8.6))

$$
\mathscr{R}^{*}=\frac{6 \pi}{25} \mathscr{H}^{2}\left(\zeta^{*} / \varphi^{*}\right) \Omega^{2} \frac{\epsilon^{*} \varphi^{* 2} a^{* 2}}{h^{* 2} \mu^{* 2} D^{* 2}} F^{* 2},
$$

wherein $\Omega$, a slowly-varying function of $h^{*} / a^{*}$, is provided in $(4.5 b)$, with $f=1$ and $t=0$ and the resistance coefficients provided in (5.9)-(5.10). Ignoring the slowly-varying dependence upon $h^{*} / a^{*}$ in these relations and noting that the external force $F^{*}$ is typically proportional to the particle volume we see that $\mathscr{R}^{*}$ essentially scales as the eighth power of $a^{*}$. For the problem of imposed shear of magnitude $G^{*}$ we have obtained (cf. (8.8))

$$
\mathscr{R}^{*}=\frac{24 \pi}{25} \mathscr{H}^{2}\left(\zeta^{*} / \varphi^{*}\right)(\mathscr{U}+\Omega)^{2} \frac{\epsilon^{*} \varphi^{* 2} a^{* 6}}{h^{*^{2}} D^{*^{2}}} G^{* 2},
$$

where $\mathscr{U}$ and $\Omega$ are again given by the mobility relations (4.5), now with the coefficients provided in (5.15)-(5.17). Here, $\mathscr{R}^{*}$ essentially scales as the sixth power of $a^{*}$.

The quadratic nonlinear dependence of the repulsive force in the 'driver' of the flow 
( $F^{*}$ in the force problem, $G^{*}$ in the second) is evident in (9.1)-(9.2). (In the dimensionless notation, the nonlinearity is manifested via the dependence upon the Péclet number.) Because of the Einstein-Smoluchowski relation, the product $\mu^{*} D^{*}$ is independent of the solution viscosity (Schnitzer \& Yariv 2014). Thus, expression (9.1) is essentially independent of the electrolyte viscosity, while expression (9.2) essentially scales as its square. The latter is qualitatively consistent with the observation of increased repulsion for highly viscous liquids (Alexander \& Prieve 1987).

It appears that the most related work in the literature is that of Tabatabaei et al. (2006), who employed the model of Cox (1997) to calculate the electroviscous forces on a sphere which is translating and rotating in the vicinity of a solid wall. While this problem is not the same as the shear-induced problem considered herein, it may nevertheless provide the requisite electroviscous forces when properly choosing the translation and rotation velocities (see $\S 5$ ). A careful scrutiny reveals that, under theses conditions, expression (9.2) coincides with the lift-force approximation derived by Tabatabaei et al. (2006). As discussed in part 1 of this sequence, an oversight in the analysis of Cox (1997) results in confusion when considering both the validity domain of his scheme and the asymptotic ordering of the different electroviscous effects; thus, apparently $O(1)$ terms is Cox's scheme turn out to be numerically large when considering moderate-Pécletnumbers conditions. Somewhat fortuitously, Cox's scheme has the same elements as in the moderate-Péclet-number model of part 2.

While the electroviscous forces are formally 'small' in the thin-double-layer limit, their irreversible nature renders them important, especially as their effect upon particle motion accumulates in time. Furthermore, these forces are amplified in the prevailing near-contact configurations, scaling inversely with the square of the gap thickness. The present illustrations thus point out to the potential importance of electroviscous forces in the behavior of colloidal dispersions. Specifically, the near-contact amplification suggests that these repulsive forces, acting to offset the London-van der Waals attraction (Israelachvili 2010), may affect such phenomena as particle coagulation, suspension stability, and particle-surface deposition. Our macroscale paradigm allows for analysis of such problems. This paradigm, moreover, may be extended to analyse streaming-potential phenomena in free-surface systems (Ohshima et al. 1984), where it is anticipated that electroviscous forces are significantly larger (Schnitzer et al. 2013).

\section{Appendix. Resistance coefficients}

It is useful to recall here the resistance relations which hold in the Stokes-flow régime when a particle undergoes a rigid-body motion in an otherwise quiescent liquid (in the absence of any electrokinetic effects). We employ the dimensionless notation of $\S 2.1$, where length variables are normalized by $a^{*}$, rectilinear and angular velocities by $v^{*}$ and $v^{*} / a^{*}$, respectively, and forces and torques by $\mu^{*} v^{*} a^{*}$ and $\mu^{*} v^{*} a^{* 2}$, respectively. In particular, we consider a spherical particle with instantaneous rectilinear velocity (of its centre) $\boldsymbol{U}$ and angular velocity $\boldsymbol{\Omega}$. The hydrodynamic force $\grave{\boldsymbol{F}}$ and torque (about the particle centre) $\grave{\boldsymbol{T}}$ are provided (Happel \& Brenner 1965) by the linear representations

$$
\grave{\boldsymbol{F}}=-F \cdot \boldsymbol{U}-M \cdot \boldsymbol{\Omega}, \quad \grave{\boldsymbol{T}}=-M^{\dagger} \cdot \boldsymbol{U}-T \cdot \boldsymbol{\Omega},
$$

where the resistance tensors $F, T$ and $M$ are functions of geometry.

In the two problems considered in this paper the geometry is specified by a sphere (the particle boundary) and a plane. It therefore provides a single fixed vector, $\hat{e}_{\perp}$, the (say unit) normal to the plane. It follows that the true tensors $F$ and $T$ must possess the 
form

$$
F=f_{\perp} \hat{\boldsymbol{e}}_{\perp} \hat{\boldsymbol{e}}_{\perp}+f_{\|}\left(I-\hat{\boldsymbol{e}}_{\perp} \hat{\boldsymbol{e}}_{\perp}\right), \quad T=t_{\perp} \hat{\boldsymbol{e}}_{\perp} \hat{\boldsymbol{e}}_{\perp}+t_{\|}\left(I-\hat{\boldsymbol{e}}_{\perp} \hat{\boldsymbol{e}}_{\perp}\right),
$$

while the pseudo-tensor $M$ is given by

$$
M=m \epsilon \cdot \hat{e}_{\perp},
$$

in which $\epsilon$ is the alternating tensor. The scalar resistance coefficients appearing in (A 2)(A 3) can depend only upon the single geometric parameter of the problem, namely the distance $\epsilon$ from the sphere to the plane.

It is convenient to decompose the pertinent vectors in the generic form $\boldsymbol{A}=\boldsymbol{A}_{\perp}+\boldsymbol{A}_{\|}$, where

$$
\boldsymbol{A}_{\perp}=\boldsymbol{A} \cdot \hat{\boldsymbol{e}}_{\perp} \hat{\boldsymbol{e}}_{\perp}, \quad \boldsymbol{A}_{\|}=\boldsymbol{A} \cdot\left(I-\hat{\boldsymbol{e}}_{\perp} \hat{\boldsymbol{e}}_{\perp}\right) .
$$

It then follows from (A 1)-(A 2) that

$$
\grave{\boldsymbol{F}}_{\perp}=f_{\perp} \boldsymbol{U}_{\perp}, \quad \grave{\boldsymbol{T}}_{\perp}=t_{\perp} \boldsymbol{\Omega}_{\perp}
$$

and

$$
\grave{\boldsymbol{F}}_{\|}=-f_{\|} \boldsymbol{U}_{\|}-m \boldsymbol{\Omega}_{\|} \times \hat{\boldsymbol{e}}_{\perp}, \quad \grave{\boldsymbol{T}}_{\|}=-t_{\|} \boldsymbol{\Omega}_{\|}+m \boldsymbol{U}_{\|} \times \hat{\boldsymbol{e}}_{\perp}
$$

\section{REFERENCES}

Alexander, B. M. \& Prieve, D. C. 1987 A hydrodynamic technique for measurement of colloidal forces. Langmuir 3 (5), 788-795.

Bike, S. G., Lazarro, L. \& Prieve, D. C. 1995 Electrokinetic lift of a sphere moving in slow shear flow parallel to a wall I. Experiment. J. Colloid Interface Sci. 175 (2), 411-421.

Bike, S. G. \& PRIEve, D. C. 1990 Electrohydrodynamic lubrication with thin double layers. J. Colloid Interface Sci. 136 (1), 95-112.

Bike, S. G. \& Prieve, D. C. 1995 Electrokinetic lift of a sphere moving in slow shear flow parallel to a wall II. Theory. J. Colloid Interface Sci. 175 (2), 422-434.

Воотн, F. 1950 The electroviscous effect for suspensions of solid spherical particles. Proc. Roy. Soc. London. A 203 (1075), 533-551.

Воотн, F. 1954 Sedimentation potential and velocity of solid spherical particles. J. Chem. Phys. 22, 1956-1968.

Bowen, W. R. \& Jenner, F. 1995 Electroviscous effects in charged capillaries. J. Colloid Interface Sci. 173 (2), 388-395.

Cooley, M. D. A. \& O'Neill, M. E. 1968 On the slow rotation of a sphere about a diameter parallel to a nearby plane wall. J. Inst. Math. Applics. 4, 163-173.

Cox, R. G. 1997 Electroviscous forces on a charged particle suspended in a flowing liquid. $J$. Fluid Mech. 338, 1-34.

Cox, R. G. \& Brenner, H. 1967 The slow motion of a sphere through a viscous fluid towards a plane surface - II. Small gap widths, including inertial effects. Chem. Engng Sci. 22, $1753-1777$.

Doi, M. \& MAKino, M. 2008 Electrokinetic boundary condition compatible with the Onsager reciprocal relation in the thin double layer approximation. J. Chem. Phys. 128, 044715.

Elton, G. A. H. 1948 Electroviscosity. I. The flow of liquids between surfaces in close proximity. Proc. Roy. Soc. London. A 194 (1037), 259.

Elton, G. A. H. 1949 Electroviscosity. III. Sedimentation phenomena in ionic liquids. Proc. Roy. Soc. London. A 197 (1051), 568-572.

Happel, J. \& Brenner, H. 1965 Low Reynolds Number Hydrodynamics. Englewood Cliffs, N. J.: Prentice-Hall.

Hinch, E. J. 1991 Perturbation Methods. Cambridge: Cambridge University Press.

Hinch, E. J. \& Sherwood, J. D. 1983 Primary electroviscous effect in a suspension of spheres with thin double layers. J. Fluid Mech. 132, 337-47.

IsRaelachVili, J. N. 2010 Intermolecular and Surface Forces. Academic Press. 
JefFrey, D. J. 1996 Some basic principles in interaction calculations. In Sedimentation of small particles in a viscous fluid (ed. E. M. Torry), chap. 4, pp. 97-124. Southampton: Computational Mechanics.

Leal, L. G. 2007 Advanced Transport Phenomena: Fluid Mechanics and Convective Transport Processes. New York: Cambridge University Press.

Lever, D. A. 1979 Large distortion of the electric double layer around a charged particle by a shear flow. J. Fluid Mech. 92 (03), 421-433.

Ohshima, H., Healy, T. W., White, L. R. \& O’Brien, R. W. 1984 Sedimentation velocity and potential in a dilute suspension of charged spherical colloidal particles. J. Chem. Soc., Faraday Trans. 80 (10), 1299-1317.

O'NeILl, M. E. 1968 A sphere in contact with a plane wall in a slow linear shear flow. Chem. Engrg Sci. 23 (11), 1293-1298.

O'NeILl, M. E. 1969 On asymmetrical slow viscous flows caused by the motion of two equal spheres almost in contact. Math. Proc. Cambridge Philos. Soc. 65 (2), 543-556.

O'Neill, M. E. \& Stewartson, K. 1967 On the slow motion of a sphere parallel to a nearby plane wall. J. Fluid Mech. 27, 705-724.

Rice, C. L. \& WhiteheAd, R. 1965 Electrokinetic flow in a narrow cylindrical capillary. The Journal of Physical Chemistry 69 (11), 4017-4024.

Russel, W. B. 1976 Low-shear limit of the secondary electroviscous effect. J. Colloid Interface Sci. 55 (3), 590-604.

Russel, W. B. 1978a Bulk stresses due to deformation of the electrical double layer around a charged sphere. J. Fluid Mech. 85 (04), 673-683.

Russel, W. B. $1978 b$ The rheology of suspensions of charged rigid spheres. J. Fluid Mech. 85 (02), 209-232.

Saville, D. A. 1977 Electrokinetic effects with small particles. Annu. Rev. Fluid Mech. 9, $321-337$.

Schnitzer, O., Frankel, I. \& Yariv, E. $2012 a$ Shear-induced electrokinetic lift at large Péclet numbers. Math. Model. Nat. Phenom. 7 (04), 64-81.

Schnitzer, O., Frankel, I. \& Yariv, E. $2012 b$ Streaming-potential phenomena in the thinDebye-layer limit. Part 2. Moderate-Péclet-number theory. J. Fluid Mech. 704, 109-136.

Schnitzer, O., Frankel, I. \& Yariv, E. 2013 Electrokinetic flows about conducting drops. J. Fluid Mech. 722, 394-423.

Schnitzer, O., Khair, A. \& YARIV, E. 2011 Irreversible electrokinetic repulsion in zeroReynolds-number sedimentation. Phys. Rev. Lett. 107, 278301.

Schnitzer, O. \& YARIV, E. 2014 Nonlinear electrophoresis at arbitrary field strengths: smallDukhin-number analysis. Phys. Fluids 26 (12), 122002.

Stigter, D. 1980 Sedimentation of highly charged colloidal spheres. J. Phys. Chem. 84 (21), $2758-2762$.

Tabatabaei, S. M., van de Ven, T. G. M. \& Rey, A. D. 2006 Electroviscous sphere-wall interactions. J. Colloid Interface Sci. 301 (1), 291-301.

van de Ven, T. G. M., Warszynski, P. \& Dukhin, S. S. 1993 Electrokinetic lift of small particles. J. Colloid Interface Sci. 157 (2), 328-331.

Warszynski, P. \& VAN DE Ven, T. G. M. 1990 Electroviscous forces. Faraday Discussions of the Chemical Society 90, 313-321.

WARsZynski, P. \& VAN DE VEN, T. G. M. 1991 Effect of electroviscous drag on the coagulation and deposition of electrically charged colloidal particles. Adv. Colloid Interface Sci. 36, 3363.

Warszynski, P., Wu, X. \& VAn DE Ven, T. G. M. 1998 Electrokinetic lift force for a charged particle moving near a charged wall - a modified theory and experiment. Colloid Surface A 140 (1-3), 183-198.

Watterson, I. G. \& White, L. R 1981 Primary electroviscous effect in suspensions of charged spherical particles. J. Chem. Soc., Faraday Trans. 277 (7), 1115-1128.

Yariv, E., Schnitzer, O. \& Frankel, I. 2011 Streaming-potential phenomena in the thinDebye-layer limit. Part 1. General theory. J. Fluid Mech. 685, 306-334. 